DOI: 10.1002/adma.((please add manuscript number))

Submitted to

Article type: Communication

\section{A selectively permeable membrane for enhancing cyclability of organic sodium-ion batteries}

Chengliang Wang, Cheng Jiang, Yang Xu, Liying Liang, Min Zhou, Jianjun Jiang, Sukhdeep Singh, Huaping Zhao, Andreas Schober and Yong Lei*

((Optional Dedication))

Dr. Y. Xu, L. Liang, Dr. M. Zhou, Dr. H. Zhao, Prof. Y. Lei

Institute of Physics \& IMN MacroNano®

Technical University of Ilmenau, Ilmenau 98693, Germany

E-mail: yong.lei@tu-ilmenau.de

Prof. C. Wang, C. Jiang, Prof. J. Jiang

School of Optical and Electronic Information

Huazhong University of Science and Technology, Wuhan 430074, China

Dr. S. Singh, Prof. A. Schober

Institute for Chemistry and Bio-Technique \& IMN MacroNano®

Technical University of Ilmenau, Ilmenau 98693, Germany 


\section{Submitted to

Organic sodium-ion batteries (OSIBs), as promising alternatives of commercial inorganic lithium-ion batteries (LIBs), have attracted more and more attention for the foreseeable largescale applications (especially the burgeoning electric vehicles and flexible and wearable electronics), because OSIBs can combine the merits of SIBs (similar electrochemical properties compared with LIBs but having environmental benignity and vast resources) $)^{[1-7]}$ and the advantages of organic materials (flexibility, natural sources, recyclability, low cost, and easily functionalized for tuning the electrochemical performance, compared with inorganic materials) $)^{[8-31]}$.

However, organic electrode materials always suffer the dissolution in the organic electrolyte. The dissolved materials (A) move to the other electrode and react directly with (or electrodeposit on ${ }^{[32]}$ ) the electrode materials (B). The "reaction-diffusion-dissolutiondiffusion" cycle will finally deplete all the material (A) even it has a very low solubility, leading to significant performance degradation. In order to improve the cyclability, methods have been adopted to alleviate the dissolution, including using poorly-soluble materials (insoluble polymers ${ }^{[33]}$ or materials with orthogonal intermiscibility ${ }^{[34]}$ in the electrolyte), mixing with more conductive materials (or binder) ${ }^{[35]}$ or surface coating of $\mathrm{Na}^{+}$-conductive protection layer (e.g. $\mathrm{Al}_{2} \mathrm{O}_{3}, \mathrm{TiO}_{2}$ ) by atomic layer deposition (ALD) ${ }^{[36]}$. Although the selection of insoluble polymers ${ }^{[33]}$ or materials with orthogonal intermiscibility ${ }^{[34]}$ in the electrolyte has been proved to be effective to enhance the cyclability due to their insolubility, this fastidious selection of electrode materials extremely limits the application of organic materials. Here, inspired by the facile deposition and function of pinhole-free polymeric dielectric layer in organic electronics, we demonstrate a novel strategy for enhancing the cyclability by preventing the further dissolution of the active materials. The strategy is proposed on the basis of two aspects: 1) researches in organic electronics have shown that a thin dielectric layer with free pinhole can be facilely obtained by using polymeric materials 


\section{ADVANCED}

through different kinds of methods (e.g. spin-coating, drop-casting and printing techniques) ${ }^{[37-}$ 39]; 2) such a pinhole-free thin film with Na-ion permeability would be able to allow the pass of Na-ions but block the active materials, providing that the thin film is Na-ion conductive or the active materials have a larger size than those of $\mathrm{Na}$ ions and the holes in the thin film. In this case, $\mathrm{Na}$ ions can pass through the membrane and the active molecules are localized at their original side even though some of them might be dissolved. In order to demonstrate our methodology, we chose 5,7,12,14-pentacenetetrone (PT, Figure 1a) as a model, which has been studied in LIBs and showed dramatically performance decay in the first 10 cycles $^{[40-41]}$ due to its slight dissolution. However, it is possible to locate PT, considering that its size (5.4 $\AA \times 13.9 \AA$, Figure $1 \mathrm{~b})^{[42]}$ is much larger than $\mathrm{Na}$ ions $(2.0 \AA)$. Two $\mathrm{Na}^{+}$-conductive materials, poly(3,4-ethylenedioxythiophene)-poly(styrene sulfonate) (PEDOT:PSS) complex and sodium carboxymethyl cellulose (CMC) were selected to provide this selectively permeable membrane. As illustrated in Figure 1c, the $\mathrm{Na}^{+}$-conductive thin film (PEDOT:PSS as an example) permits the pass of sodium ions (visually described by using holes), while the molecules of the active materials are blocked even some of the molecules are dissolved in the electrolyte. It is worth noting that the thin film is essential to permit the pass of sodium-ions and hence such selectively permeable film is similar but not equal to the separator.

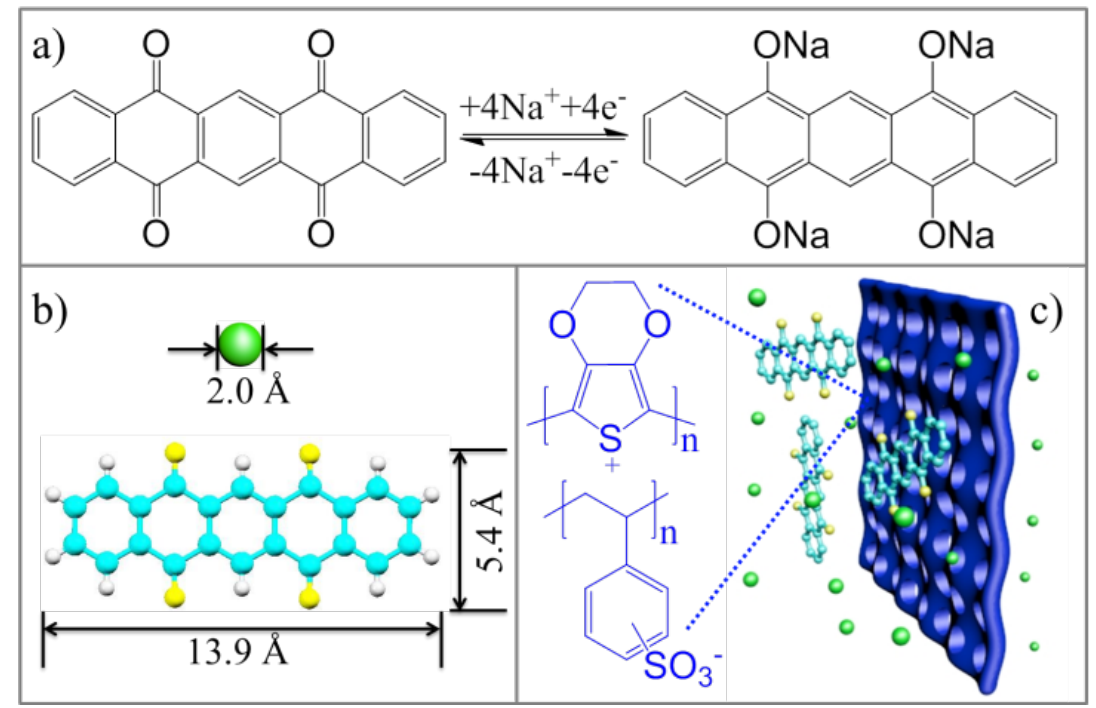




\section{ADVANCED}

Figure 1. a) Reversible Na-ion insertion/extraction mechanism of PT; b) the size of Na ions and PT molecules; c) schematic diagram of Na-ion permeable membrane, which permits the pass of $\mathrm{Na}$ ions and prevents the pass of active molecules. Inset: the chemical structure of the $\mathrm{Na}^{+}$-conductive material, PEDOT:PSS. The holes in the thin film are to show the transmission of $\mathrm{Na}$ ions visually.

The potential application of PT for OSIBs was first studied by using a half cell with sodium metal as the counter electrode. The PT electrodes were obtained by mixing PT with carbon black (Super-P) as conductive additive and poly(vinylidenedifluoride) (PVDF) or CMC as binder. Figure 2a shows the typical cyclic voltammograms (CV) of PT electrodes, which was obtained by mixing with super-P, PEDOT:PSS and CMC at a weight ratio of 70:15:10:5. The CV curves of PT electrodes mixed with super-P and other binders (e.g. PVDF or CMC individually) at different ratios did not show obvious differences in the shapes and redox potentials (Figure S1 and S2). All of them show three pair redox peaks located at $\approx 1.8,2.1$ and $2.4 \mathrm{~V}$, respectively. The two redox peaks at lower potential are reversible with almost symmetric anodic and cathodic peaks, identical currents, and very small reduction/oxidation potential gaps ( 0.04 and $0.02 \mathrm{~V}$ respectively). The redox peak at the highest potential is quasireversible, with equivalent anodic and cathodic peak areas, although the currents are not identical and the potential gap is larger $(0.2 \mathrm{~V})$. These phenomena are quite similar to the electrochemical performance of PT in lithium-ion batteries ${ }^{[40,43]}$. Accordingly, we can conclude that the three redox peaks reveal a four-electron redox reaction, as shown in Figure 1a. The first two peaks reflect a single-electron redox reaction for each of them. The third redox peak characterizes a two-electron redox reaction, which has a peak area equal to the sum of the two redox peaks at lower potential.

As mentioned above, the problem that PT electrode encounter is that PT is soluble in the organic electrolyte (propylene carbonate (PC) as the solvent in this work). As shown in Figure 


\section{Submitted to

$2 \mathrm{a}$ and S3, the current becomes decreasing after the second cycle. In order to reduce its dissolution and thereby enhance the cyclability of the batteries, kinds of traditional methods are tested firstly. However, all of them showed unsuccessful or unsatisfied enhancement, as shown in Figure 2c, 2d and Table S1: 1) Increasing the content of conductive materials as for samples PVDF-70\%-1 and PVDF-50\% (the content of PVDF binder is kept as 10\%) is helpful to improve the cyclability because of the alleviation of dissolution and the improved charge transport (due to the insulating property of $\mathrm{PT}^{[37,44]}$, higher content of PT (e.g. $>70 \%$ ) results in failure of batteries). The reason that the capacity is increasing in the first several cycles is probably due to the large particle size of PT (Figure S4) and the gradual activation of bulk PT. However, the capacity decreased rapidly afterwards, to $19.9 \mathrm{mAh} \mathrm{g}^{-1}$ after 100 cycles. This improvement is quite limited. Moreover, low content of active materials is undesirable for practical application. 2) Increasing the content of binder from $10 \%$ to $15 \%$ for samples PVDF-70\%-1 and PVDF-70\%-2 leads to dramatic performance decay, due to the insulating property ${ }^{[17,34]}$ of PVDF to $\mathrm{Na}$ ions. 3) Our previous works ${ }^{[17,34]}$ have shown that the utilization of $\mathrm{CMC}$ is useful to enhance the capability and cyclability. However, such strategy is inadequate to PT electrodes. The samples using CMC binder (CMC-50\%) instead of PVDF (PVDF-50\%) show even worse cyclability than those based on PVDF binder. The reason probably can be ascribed to the insolubility of PT and the high solubility of the previously reported materials in water for preparation of CMC-based samples. 4) Researches have shown that PEDOT:PSS surface coating is effective to confine the active materials from expansion upon cycling for $\mathrm{Li} / \mathrm{S}$ batteries ${ }^{[45]}$ and prevent the dissolution through formation of noncovalent $\mathrm{S} \cdots \mathrm{S}$ interactions between the active material and PEDOT:PSS for LIBs ${ }^{[46]}$. Although such interactions do not exist in our systems (no S atoms in PT molecules) and water is still used as the solvent for preparation, the introducing of PEDOT:PSS (PP-CMC$70 \%$ ) indeed slightly enhanced the cyclability of PT, compared with the samples with same or similar contents of conductive additive and binder (PVDF-70\%-1 and PVDF-70\%-2). This is 
probably due to the high electric conductivity and $\mathrm{Na}^{+}$-conductivity of PEDOT:PSS (see below). However, the capacity kept on decreasing to $7.5 \mathrm{mAh} \mathrm{g}^{-1}$ after 60 cycles. This can be ascribed to the incomplete encapsulation of the active materials by the binder, which is typical in traditional mixing methods. 5) The ALD surface coating is capable of complete encapsulation and has proved to be effective for many inorganic materials for maintaining the structural integrity, inhibiting the chemical reactions, obstructing the electrons to decompose the electrolyte and preventing the dissolution of the active materials ${ }^{[47]}$. The ALD coating of 50 cycles $(2.5 \mathrm{~nm})$ of $\mathrm{TiO}_{2}$ indeed showed a slight improvement of cyclability. However, such enhancement is also disappointing and the increase in the capacity after $\mathrm{TiO}_{2}$ coating is partially attributed to the $\mathrm{Na}^{+}$-intercalation ${ }^{[47]}$ of $\mathrm{TiO}_{2}$. The capacity decreased from $109 \mathrm{mAh}$ $\mathrm{g}^{-1}$ (for the fifth cycle) to $13 \mathrm{mAh} \mathrm{g}^{-1}$ after 100 cycles. This may be due to the lowtemperature deposition of $\mathrm{TiO}_{2}$ which leads to poor continuity of $\mathrm{TiO}_{2}$ and invalid protection.
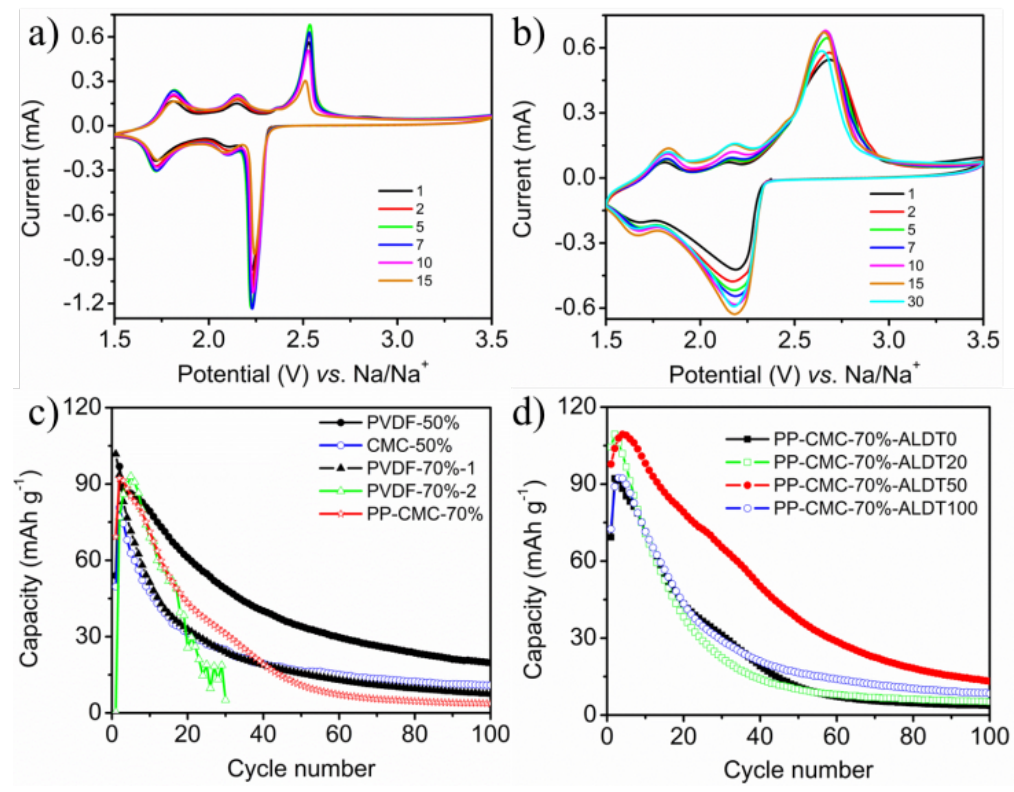

Figure 2. Typical CV curves of PT films. The batteries were assembled a) without and b) with PPS membrane at a scan rate of $0.5 \mathrm{mV} \mathrm{s}^{-1}$. c) Cycling performance of PT. The percentage shown in the Figure is the content of the active materials PT. The percentage of the binder is kept as $10 \%$ for the first three samples and $15 \%$ for the sample PVDF- $70 \%-2$. The percent-ages of PEDOT:PSS (abbreviated as PP) and CMC in the sample PP-CMC-70\% 
are $10 \%$ and $5 \%$ respectively. d) The effect of ALD coating of $\mathrm{TiO}_{2}$. For example, ALDT20 stands for ALD coating of $\mathrm{TiO}_{2}$ with 20 cycles (ca. $1 \mathrm{~nm}$ ).

The fruitless attempts of aforementioned traditional methods urge us to search novel strategy for enhancing the cyclability. The reason can be ascribed to the incomplete encapsulation of all these methods. Therefore, inspired by the facile deposition and function of pinhole-free polymeric dielectric layer in organic electronics, a pinhole-free thin film would be able to permit the pass of $\mathrm{Na}$ ions but block the active materials, providing that the thin film is Na-ion conductive or the active materials have a larger size than that of $\mathrm{Na}$ ions and the holes in the thin film. Based on the above results, PEDOT:PSS and CMC were chosen as the materials for the selectively permeable thin film because of their high Na-ion conductivities, while PVDF is insulating for $\mathrm{Na}$ ions and therefore is not appropriate. In order to simplify the fabrication process, drop-casting method ${ }^{[48-49]}$ was used to deposit PEDOT:PSS or CMC onto one side of the separator (glass fiber, Figure S5). The glass fiber was placed on a substrate which has been previously modified by hydrophobic self-assembly monolayers, leading to a deposition of the selectively permeable thin film only on one side of the separator. The partial penetration of the solution into the porous separator is conducive to the formation of thin film on the separator (Figure S5 and S6). It should be noted that other methods (e.g. spin-coating, dip-coating or printing etc.) are also possible to fabricate this selectively permeable thin film for reducing the cost. The effect of this thin film was investigated by using the batteries obtained under same conditions as above. By applying PPS (PEDOT:PSS-treated separator) in the battery, the current keeps almost stable in the first 50 cycles (Figure $2 \mathrm{~b}$ and S3), compared with the continuing decline after the second cycle in the samples without PPS (Figure 2a and S3). The slight shift of the redox peaks (larger potential gap between the oxidation peak and the reduction peak) and the merging of the second and the third reduction peak suggest the blocking effect of the pinhole-free film to the electrolyte. 
Sum significant cyclability enhancement As expected, after application of PPS, the samples show significant cyclability enhancement (Figure 3a, S7 and Table S1). Without PPS, the capacity decreased to $10.9 \mathrm{mAh} \mathrm{g}^{-1}$ after 50 cycles and $2.4 \mathrm{mAh} \mathrm{g}^{-1}$ after 300 cycles for sample PP-CMC-70\%; however, with PPS, the capacity kept stable after 70 cycles and maintained at $73 \mathrm{mAh} \mathrm{g}^{-1}$ after 300 cycles. Such enhancement is effective to all the PT electrodes mixed with different binders at different contents (e.g. PVDF-70\%-1 in Figure 3a and S7 or PVDF-50\% in Figure S8). Considering the low contents and low capacities of Super-P and PPS (Figure S9 and S10), the capacity contribution from them are negligible. In other words, the remaining capacity mostly comes from the un-dissolved PT. The decrease of the capacity from 30 to 70 cycles is probably due to the gradual dissolution of PT in the electrolyte. The high stability afterwards suggests that PT becomes saturated in the electrolyte in this side of PPS (Figure S11). The PPS blocks the movement of PT to the anode side, and thereby prevents the direct reaction between PT and $\mathrm{Na}$ and further dissolution of PT, leading to significant improvement of the cyclability. Without PPS, PT will be dissolved gradually and the direct reaction will deplete all the PT molecules, resulting in deactivated battery finally. The slight shift of the charging/discharging plateau (Figure 3b, similar to the $\mathrm{CV}$ curves) and the decay under fast-charge and fastdischarge process (Figure 3c) further proved the blocking effect of PPS membrane. Considering that the rate performance is not significantly affected by the ionic insulating glass fibre with large interspacing pores and the capacities and cyclability of the samples with PPS under slow charging/discharging rate are much higher than those without PPS, therefore the blocking effect rather than the ionic insulating property of the membrane is the dominated role leading to low capacity under high rate. Additionally, compared with the importance of long lifetime, the charging rate $\left(50,100\right.$ and $\left.200 \mathrm{~mA} \mathrm{~g}^{-1}\right)$ is acceptable, corresponding to an adequate charging time around $2.5,1$, and $0.5 \mathrm{~h}$ respectively. The further application of ALD surface coating of the electrodes didn't show further improvement of the cyclability of the batteries (Figure 3d), which probably can be ascribed to the low-temperature deposition, 
forming low film quality. The inactive and insulating protective $\mathrm{Al}_{2} \mathrm{O}_{3}$ layer leads to an even lower capacity. These results reconfirmed that ALD coating is inapplicable to our systems and our novel method is the best approach to enhance the cyclability. Additionally, all these samples also show slow performance decay after $\sim 80$ cycles, which again supported the effective blocking effect of PPS membrane. The samples with CS (CMC-treated separator) also show clear cyclability improvement as for different electrodes (Figure 3a, S7, S12), suggesting the enhancement is due to the blocking effect rather than the material adopted. The weaker enhancement of the CS compared with PPS probably can be ascribed to the higher flexibility and conductivity of PEDOT:PSS than those of CMC. Due to the sulfonate functional groups, the component PSS in PPS shows a strong ion exchange property of sodium ions and thereby has been used for water softening ${ }^{[50]}$. Such high Na-ion conductivity also contributed to the better enhancement effect of PPS. On the other hand, a thick film of CMC (see supporting information, Figure S13) can thoroughly block the pass of $\mathrm{Na}$ ions and lead to failure of the batteries, suggesting the lower Na-ion conductivity of CMC and the effect of this thin film to prevent the movement of the active materials. All of these results show that the strategy using selectively permeable membrane to prevent the movement of active materials to the other side of the battery is successful. There is no special requirement for the active materials and such strategy can be extended to many anode and cathode materials. 

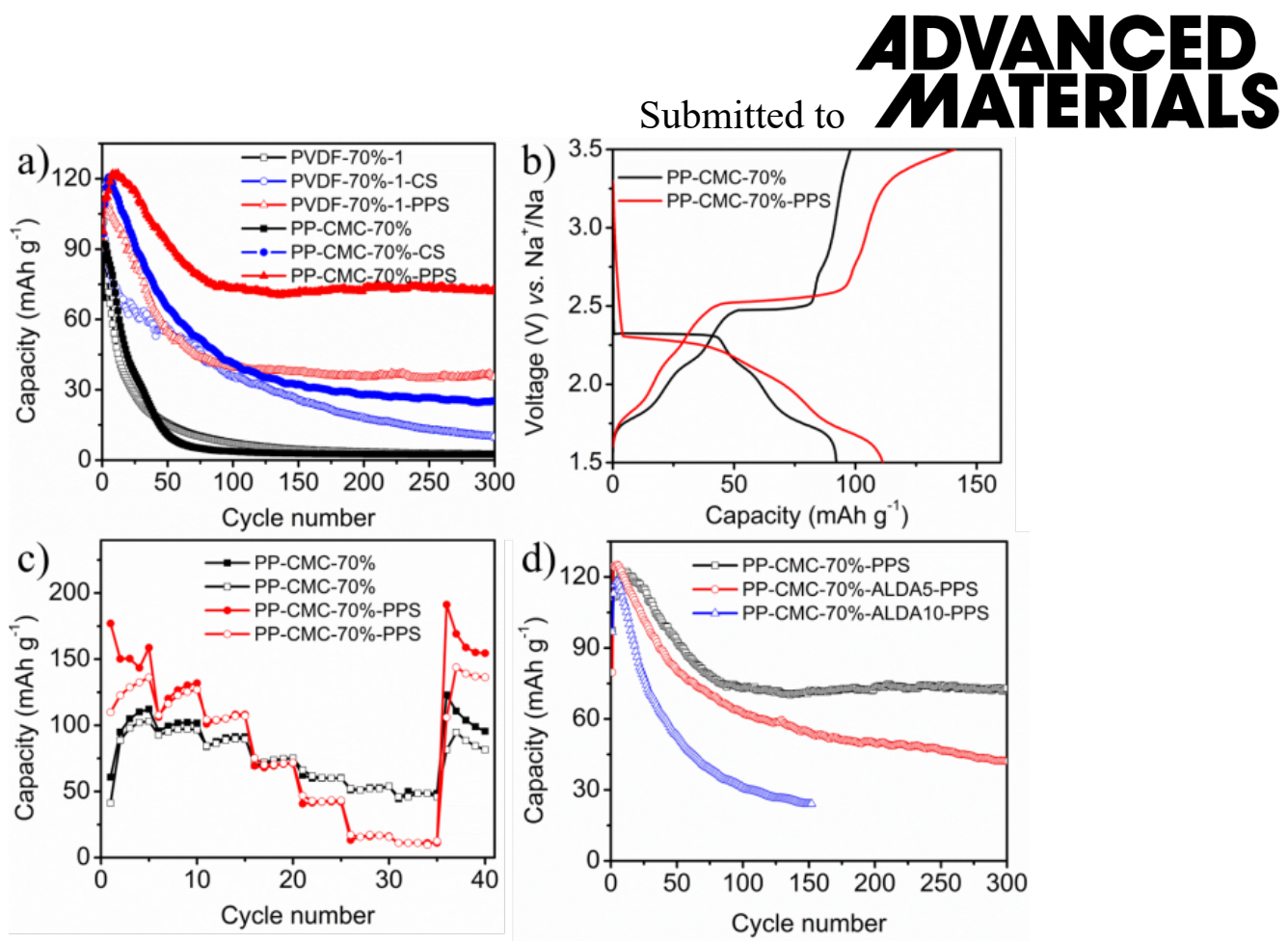

Figure 3. a) Significant enhancement of cycling performance of PT electrodes by using CS and PPS. b-d) Electrochemical performances of PT films mixed with super-P, PEDOT:PSS and $\mathrm{CMC}$ at a ratio of 70:15:10:5. b) Voltage profiles of the second cycle at a current density of $50 \mathrm{~mA} \mathrm{~g}^{-1}$; c) rate cyclability (left to right: 50,100, 200,1000, 2000, 5000 to $50 \mathrm{~mA} \mathrm{~g}{ }^{-1}$ ). Black: without and Red: with PPS. Solid symbol: charge, open symbol: discharge. d) The effect of ALD coating of $\mathrm{Al}_{2} \mathrm{O}_{3}$ on the cycling performance of PT with PPS. For example, ALDA5 stands for ALD coating of $\mathrm{Al}_{2} \mathrm{O}_{3}$ with 5 cycles (ca. $0.5 \mathrm{~nm}$ ).

The generality of this strategy is performed by using disodium rhodizonate salt (DSR) as a control experiment. DSR has been reported for SIBs, which can only be cycled under 3 V (vs. $\mathrm{Na} / \mathrm{Na}^{+}$) due to the dissolving of its oxidation state around $3.2 \mathrm{~V}$ (Figure S14) ${ }^{[25,34]}$. However, after application of PPS, the cyclability of DSR electrodes cycled up to $3.5 \mathrm{~V}$ can also be significantly improved (Figure S15). These results indicate the universality of our novel strategy. It should be noted that the direct coating of this thin film on the electrodes will lead to much lower performance decay in the first several cycles. Compared with all the traditional methods, this selectively permeable film can provide full encapsulation of the electrodes, similar to the ALD protective layer. However, compared with ALD deposition, this technique 
has the advantages of facial fabrication, possibility to be obtained in large area and low cost.

Such selectively permeable film is extremely suitable for organic electrodes which can't undergo high temperature for achieving full encapsulation by ALD. Additionally, a more insoluble electrode material will definitely show even better performance cyclability, especially for long-term cycling. However, such long-term cyclability can't be achieved for traditional batteries, due to the nonstop "reaction-diffusion-dissolution-diffusion" cycle which will deplete all the materials even the material has a very low solubility. The further improvement of the selectively permeable films and the subsequent cyclability are underway.

In conclusion, for the first time, we have studied the electrochemical performance of PT for organic sodium-ion batteries. Inspired by the pinhole-free dielectric films in organic electronics, we developed a novel strategy for enhancing the cyclability of the batteries by preventing the further dissolution of the active materials. The application of a selectively permeable membrane is proved to be effective to allow the pass of $\mathrm{Na}$ ions but block the active molecules. By applying PPS to assemble the battery, the capacity of PT after 300 cycles was improved from 2.4 to $73 \mathrm{mAh} \mathrm{g}^{-1}$. Compared with all the traditional methods, this selectively permeable film can provide full encapsulation of the electrodes, which is extremely suitable for organic electrode materials. There is no special requirement for the active materials and hence our work will tremendously extend the application of many materials for organic sodium-ion batteries. Moreover, the size of Li ions is smaller than $\mathrm{Na}$ ions and therefore this strategy would be also applicable to lithium-ion batteries. Such strategy of using selectively permeable membrane opens a new path for enhancing the cyclability and can also be extended to other energy storage devices, which will pave a venue to practical applications of OSIBs for foreseeable large-scale and wearable electronics.

\section{Supporting Information}

Supporting Information is available online from the Wiley Online Library or from the author. 


\section{Acknowledgements}

C. Wang would like to acknowledge the financial support from National 1000-Talents Program. The authors acknowledge the financial support from European Research Council (ThreeDsurface: 240144), BMBF (ZIK-3DNanoDevice: 03Z1MN11), BMBF (Meta-ZIKBioLithoMorphie: 03Z1M512), German Research Foundation (DFG: LE 2249_4-1) and National Natural Science Foundation of China (51203067).

Received: ((will be filled in by the editorial staff))

Revised: ((will be filled in by the editorial staff))

Published online: ((will be filled in by the editorial staff)) 
Submitted to

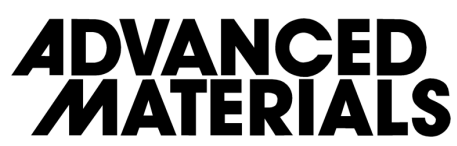

[1] N. Yabuuchi, K. Kubota, M. Dahbi, S. Komaba, Chem. Rev. 2014, 114, 11636.

[2] S.-W. Kim, D.-H. Seo, X. Ma, G. Ceder, K. Kang, Adv. Energy Mater. 2012, 2, 710.

[3] V. Palomares, M. Casas-Cabanas, E. Castillo-Martinez, M. H. Han, T. Rojo, Energy Environ. Sci. 2013, 6, 2312.

[4] M. D. Slater, D. Kim, E. Lee, C. S. Johnson, Adv. Funct. Mater. 2013, 23, 947.

[5] L. Liang, Y. Xu, C. Wang, L. Wen, Y. Fang, Y. Mi, M. Zhou, H. Zhao, Y. Lei, Energy Environ. Sci. 2015, 8, 2954.

[6] H. Pan, Y.-S. Hu, L. Chen, Energy Environ. Sci. 2013, 6, 2338.

[7] Y. Xu, M. Zhou, X. Wang, C. Wang, L. Liang, F. Grote, M. Wu, Y. Mi, Y. Lei, Angew. Chem. Int. Ed. 2015, 54, 8768.

[8] B. Häupler, A. Wild, U. S. Schubert, Adv. Energy Mater. 2015, 5, 1402034.

[9] Z. Song, H. Zhou, Energy Environ. Sci. 2013, 6, 2280.

[10] Y. Liang, Z. Tao, J. Chen, Adv. Energy Mater. 2012, 2, 742.

[11] M. Armand, S. Grugeon, H. Vezin, S. Laruelle, P. Ribiere, P. Poizot, J. M. Tarascon, Nature Mater. 2009, 8, 120.

[12] T. Nokami, T. Matsuo, Y. Inatomi, N. Hojo, T. Tsukagoshi, H. Yoshizawa, A. Shimizu, H. Kuramoto, K. Komae, H. Tsuyama, J.-i. Yoshida, J. Am. Chem. Soc. 2012, 134, 19694.

[13] W. Walker, S. Grugeon, O. Mentre, S. Laruelle, J.-M. Tarascon, F. Wudl, J. Am. Chem. Soc. 2010, 132, 6517.

[14] P. Sharma, D. Damien, K. Nagarajan, M. M. Shaijumon, M. Hariharan, J. Phys. Chem. Lett. 2013, 4, 3192.

[15] C. Luo, R. Huang, R. Kevorkyants, M. Pavanello, H. He, C. Wang, Nano Lett. 2014, 14, 1596.

[16] Y. Liang, P. Zhang, S. Yang, Z. Tao, J. Chen, Adv. Energy Mater. 2013, 3, 600. 
[17] C. Wang, Y. Xu, Y. Fang, M. Zhou, L. Liang, S. Singh, H. Zhao, A. Schober, Y. Lei, J. Am. Chem. Soc. 2015, 137, 3124 .

[18] A. Abouimrane, W. Weng, H. Eltayeb, Y. Cui, J. Niklas, O. Poluektov, K. Amine, Energy Environ. Sci. 2012, 5, 9632.

[19] Y. Park, D.-S. Shin, S. H. Woo, N. S. Choi, K. H. Shin, S. M. Oh, K. T. Lee, S. Y. Hong, Adv. Mater. 2012, 24, 3562.

[20] L. Zhao, J. Zhao, Y.-S. Hu, H. Li, Z. Zhou, M. Armand, L. Chen, Adv. Energy Mater. 2012, 2, 962 .

[21] S. Wang, L. Wang, Z. Zhu, Z. Hu, Q. Zhao, J. Chen, Angew. Chem. Int. Ed. 2014, 53, 5892.

[22] H. Chen, M. Armand, M. Courty, M. Jiang, C. P. Grey, F. Dolhem, J.-M. Tarascon, P. Poizot, J. Am. Chem. Soc. 2009, 131, 8984.

[23] A. Vlad, K. Arnould, B. Ernould, L. Sieuw, J. Rolland, J.-F. Gohy, J. Mater. Chem. A 2015, 3, 11189.

[24] Z. Song, Y. Qian, T. Zhang, M. Otani, H. Zhou, Adv. Sci. 2015, 2, 1500124.

[25] K. Chihara, N. Chujo, A. Kitajou, S. Okada, Electrochim. Acta 2013, 110, 240.

[26] W. Luo, M. Allen, V. Raju, X. Ji, Adv. Energy Mater. 2014, 4, 1400554.

[27] W. Deng, X. Liang, X. Wu, J. Qian, Y. Cao, X. Ai, J. Feng, H. Yang, Sci. Rep. 2013, 3, 2671.

[28] K. Sakaushi, E. Hosono, G. Nickerl, T. Gemming, H. Zhou, S. Kaskel, J. Eckert, Nature Commun. 2013, 4, 1485.

[29] E. Castillo-Martínez, J. Carretero-González, M. Armand, Angew. Chem. Int. Ed. 2014, $53,5341$.

[30] M. Yao, K. Kuratani, T. Kojima, N. Takeichi, H. Senoh, T. Kiyobayashi, Sci. Rep. 2014, 4, 3650.

[31] K. Zhang, C. Guo, Q. Zhao, Z. Niu, J. Chen, Adv. Sci. 2015, 2, 1500018. 
[32] B. Xiao, J. Liu, Q. Sun, B. Wang, M. N. Banis, D. Zhao, Z. Wang, R. Li, X. Cui, T.-K. Sham, X. Sun, Adv. Sci. 2015, 2, 1500022.

[33] H.-g. Wang, S. Yuan, D.-1. Ma, X.-1. Huang, F.-1. Meng, X.-b. Zhang, Adv. Energy Mater. 2014, 4, 1301651.

[34] C. Wang, Y. Fang, Y. Xu, L. Liang, M. Zhou, H. Zhao, Y. Lei, Adv. Funct. Mater. 2016, 26, 1777.

[35] P. Bu, S. Liu, Y. Lu, S. Zhuang, H. Wang, F. Tu, Int. J. Electrochem. Sci 2012, 7, 4617.

[36] C. Luo, J. Wang, X. Fan, Y. Zhu, F. Han, L. Suo, C. Wang, Nano Energy 2015, 13, 537.

[37] C. Wang, H. Dong, W. Hu, Y. Liu, D. Zhu, Chem. Rev. 2012, 112, 2208.

[38] M.-H. Yoon, H. Yan, A. Facchetti, T. J. Marks, J. Am. Chem. Soc. 2005, 127, 10388.

[39] D. Ji, L. Jiang, Y. Guo, H. Dong, J. Wang, H. Chen, Q. Meng, X. Fu, G. Tian, D. Wu, G. Yu, Y. Liu, W. Hu, Adv. Funct. Mater. 2014, 24, 3783.

[40] M. Yao, S.-i. Yamazaki, H. Senoh, T. Sakai, T. Kiyobayashi, Mater. Sci. Eng. B 2012, $177,483$.

[41] M. Yao, H. Senoh, T. Sakai, T. Kiyobayashi, Int. J. Electro. Sci 2011, 6, 2905.

[42] D. Käfer, M. El Helou, C. Gemel, G. Witte, Cryst. Growth Des. 2008, 8, 3053.

[43] J. E. Almlof, M. W. Feyereisen, T. H. Jozefiak, L. L. Miller, J. Am. Chem. Soc. 1990, $112,1206$.

[44] O. D. Jurchescu, M. Popinciuc, B. J. v. Wees, T. T. M. Palstra, Adv. Mater. 2007, 19, 688.

[45] Y. Yang, G. Yu, J. J. Cha, H. Wu, M. Vosgueritchian, Y. Yao, Z. Bao, Y. Cui, ACS Nano 2011, 5, 9187.

[46] T. Ma, Q. Zhao, J. Wang, Z. Pan, J. Chen, Angew. Chem. Int. Ed. 2016, 55, 6428.

[47] X. Meng, X.-Q. Yang, X. Sun, Adv. Mater. 2012, 24, 3589. 


\section{ADNANEDS
MAAERRALIS}

[48] C. Wang, Y. Liu, Z. Ji, E. Wang, R. Li, H. Jiang, Q. Tang, H. Li, W. Hu, Chem. Mater. 2009, 21, 2840.

[49] C. Wang, Y. Liu, Z. Wei, H. Li, W. Xu, W. Hu, Appl. Phys. Lett. 2010, 96, 143302.

[50] K.-M. Mangold, C. Weidlich, J. Schuster, K. Jüttner, J. Appl. Electrochem. 2005, 35, 1293. 